Documentation et bibliothèques

DOCUMENTATION BIBLIOTHEQUES

\title{
L'élagage : une fonction de la bibliothèque encore à inventer
}

\section{Clément Tremblay}

Volume 22, numéro 1, mars 1976

URI : https://id.erudit.org/iderudit/1055334ar

DOI : https://doi.org/10.7202/1055334ar

Aller au sommaire du numéro

\section{Éditeur(s)}

Association pour l'avancement des sciences et des techniques de la documentation (ASTED)

\section{ISSN}

0315-2340 (imprimé)

2291-8949 (numérique)

Découvrir la revue

Citer cet article

Tremblay, C. (1976). L'élagage : une fonction de la bibliothèque encore à inventer. Documentation et bibliothèques, 22(1), 9-15.

https://doi.org/10.7202/1055334ar

\section{Résumé de l'article}

La chaîne documentaire n'est vraiment complète qu'au moment où elle fait une place importante à l'élagage. Cependant, tout porte à croire que l'élagage demeure l'aspect négligé de nos bibliothèques d'enseignement et de recherche malgré une pression de plus en plus forte d'une documentation en croissance continuelle. Il devient alors urgent de développer une véritable fonction d'élagage qui tienne compte aussi bien des difficultés rencontrées jusqu'à maintenant dans son instauration que des possibilités de coopération offertes par les bibliothèques dites de conservation.
Tous droits réservés (C) Association pour l'avancement des sciences et des techniques de la documentation (ASTED), 1976
Ce document est protégé par la loi sur le droit d'auteur. L’utilisation des services d'Érudit (y compris la reproduction) est assujettie à sa politique d'utilisation que vous pouvez consulter en ligne.

https://apropos.erudit.org/fr/usagers/politique-dutilisation/ 


\title{
L'élagage: une fonction de la bibliothèque encore à inventer
}

\author{
Clément Tremblay \\ Développement des collections \\ Bibliothèque \\ Université du Québec à Rimouski
}

La chaîne documentaire n'est vraiment complète qu'au moment où elle fait une place importante à l'élagage. Cependant, tout porte à croire que l'élagage demeure l'aspect négligé de nos bibliothèques d'enseignement et de recherche malgré une pression de plus en plus forte d'une documentation en croissance continuelle. Il devient alors urgent de développer une véritable fonction d'élagage qui tienne compte aussi bien des difficultés rencontrées jusqu'à maintenant dans son instauration que des possibilités de coopération offertes par les bibliothèques dites de conservation.

The sequence of documentation may not be considered complete until weeding is given due consideration. However, in all likelihood, weeding remains a neglected consideration of our teaching and research libraries in spite of pressure from ever-increasing documentation. It is therefore becoming urgent to implement true weeding duties to deal both with the problems encountered to date in the setting up process, and the possible cooperation from libraries dedicated to preservation.

El entresacamiento tiene un sitio muy importante en la cadena documental. Sin embargo, ha sido un aspecto desatendido de nuestras bibliotecas de enseñanza y de busca a pesar del crecimiento continual de la documentación que ejercia una presión muy fuerte. El desarrollo de la nueva función se ve muy urgente viendo leas dificultades encontradas hasta ahora en su instauración, y las posibilidades de cooperación de parte de las bibliotecas de conservación.

«Toute bonne université doit s'enrichir constamment, sinon ses collections deviennent désuètes et perdent de leur intérêt et de leur valeur. On peut comparer la bibliothèque à un organisme vivant qui s'alimente de nouvelles acquisitions et qui dépérit si cette nourriture lui manque.»'

Cette image évoquée par les auteurs du rapport DOWNS reflète à merveille la situation de nos bibliothèques lorsqu'elles sont privées d'un budget régulier de développement des collections. Elle s'avère cependant fort incomplète. Non seulement les bibliothèques doivent s'alimenter de nouvelles acquisitions

1. Robert Downs, Ressources des bibliothèques d'université et de recherche au Canada, Ottawa, AUCC, 1967, p. 230. pour ne pas souffrir d'inanition, mais encore elles doivent être en mesure de rejeter les masses de documents devenus inutiles pour ne pas mourir d'une trop forte réplétion. Or, de façon générale, les bibliothèques d'enseignement et de recherche n'ont pas de système rénal pour assurer la sélection des éléments à conserver et à rejeter ${ }^{2}$.

2. Nous nous limitons, dans le cadre de cet article, aux bibliothèques d'enseignement et de recherche et à la documentation imprimée. Toutefois, il est certain que le problème se pose pour d'autres types de bibliothèques et, en particulier, pour les bibliothèques publiques. Dans ce dernier cas, le problème est ressenti avec une certaine acuité puisqu'elles doivent suivre l'actualité de plus près. Par ailleurs, la documentation audiovisuelle est également soumise aux lois de l'élagage étant donné qu'elle est sujette, dans une large mesure, à un vieillissement très rapide. 


\section{Silence de la documentation professionnelle}

Si la documentation professionnelle abonde en ce qui concerne les fonctions premières de la bibliothèque que sont le choix, le traitement, la conservation et la diffusion, elle se fait, par contre, pratiquement silencieuse sur l'élagage et l'élimination de la documentation impertinente. Sous la rubrique "Discording of books, periodicals, etc.", la revue Library Literature signale tout au plus une douzaine d'articles pour les années 1972 et 1973. Elle n'est guère plus loquace au sujet de l'obsolescence, des problèmes d'espace ou d'évaluation des collections, questions reliées d'assez près au sujet qui nous occupe. Cela est d'autant plus inquiétant que la même revue regorge de références à des articles sur l'obscénité, la pornographie, la censure et autres questions névralgiques pour l'avenir des sciences de la documentation. Par ailleurs, des manuels aussi classiques que ceux de G. P. Lyle, The Administration of College Library et de R. D. Rogers et D. Weber, University Library Administration se font remarquer par la rapidité qu'ils manifestent à escamoter le sujet. C'est à croire que cette notion est inexistante dans notre vocabulaire professionnel ${ }^{3}$.

\section{Absence d'actions concrètes}

Les gestes concrets consacrés à l'élagage des collections d'une bibliothèque semblent également le fait de rares exceptions. II est vrai que les tentatives en ce sens qu'ont entreprises certaines bibliothèques québécoises ont connu une telle publicité négative que plus d'un bibliothécaire va y penser très sérieuse-

3. Nous pouvons facilement réduire la bibliographie sur le sujet à trois références principales:

a) Cooper, Marianne. "Criteria for weeding of collections", Library Resources and Technical Services, vol. 12 , no. 3 (1968), 339-351. Une approche à la fois théorique et pratique de critères relatifs à l'élagage.

b) Leimkuhler, Ferdinand $F$. "Storage policies for information systems", in Mackenzie, A. Graham and Stuart, Ian M., (Eds.) Planning Library Services, Lancaster, University of Lancaster, 1969. (Occasional Papers, no. 3). Une étude des coûts d'acquisition, de conservation et d'utilisation de la documentation. Détermine la période de conservation préalable à l'élagage pour laquelle le coût moyen de chaque utilisation est minimal.

c) Sinha, Banik. Operations Research in Controlled Acquisition and Weeding of Library Collection. Philadelphia, University of Pennsylvania, 1971, (Ph. D. Dissertation in operation research). L'auteur décrit un modèle de développement d'une collection de bibliothèque qui veut permettre une circulation maximale de la documentation tout en tenant compte des problèmes d'espace et de budget. ment avant d'amorcer pareille démarche. De plus, les organigrammes administratifs comme les descriptions de tâches ne font à peu près pas mention de cette fonction. Le comité sur les tâches professionnelles de la Corporation des bibliothécaires professionnels du Québec retient, parmi 59 tâches considérées importantes, une seule tâche concernant l'élagage. Et encore est-elle reliée à la microreproduction, comme si elle n'était pas suffisamment valable pour justifier qu'on lui réserve toute une ligne. Bien que faisant partie intégrante d'un système documentaire, l'élagage occupe donc une place si marginale dans la description du système que nous pouvons facilement conclure, sans trop risquer de faire erreur, que l'activité correspondante doit être, elle aussi, très marginale.

II semble donc que nous soyons placés devant un fait: nous accumulons la documentation sans pour autant être assurés que nous enrichissons nécessairement nos collections. Pourtant, la situation devient de plus en plus difficile au fur et à mesure qu'elle devient incontrôlable.

\section{Une situation d'urgence}

Point n'est besoin d'élaborer longuement sur l'explosion actuelle de l'information. Le problème est suffisamment connu. Rappelons-en seulement ici quelques facettes qui soulignent l'impact, sur les bibliothèques, de cette croissance "sauvage» de l'information documentaire.

II apparaît de plus en plus clairement que l'accroissement de l'édition scientifique, à savoir celle qui fait l'objet de la grande part des acquisitions de nos bibliothèques d'enseignement et de recherche, suit à peu près le même rythme de progression que l'accroissement de la consommation d'énergie, pour aboutir probablement au même cul-de-sac. La bibliothèque de l'université Columbia a mis 192 ans (1754-1946) à acquérir son premier million de livres, mais elle ne mettra que 14 ans pour son deuxième million et assurément moins d'années encore pour acquérir ses troisième et quatrième millions ${ }^{4}$. L'université John Hopkins, de son côté, fondée en 1876, possédait 100,000 livres en 1900, 1,5 million de volumes en 1970 et, au rythme de plus de 100,000 nouveaux volumes par année, escomptait en avoir 2,5 millions en 1976, année de son cen-

4. Chiffres cités dans C.B. Weinberg, "The university library: analysis and proposals», Management Science, vol. 21 , no. 2 (October 1974), 130. 
tenaire ${ }^{5}$. Conséquence immédiate de cette surconsommation: nous nous dirigeons directement vers un engorgement des bibliothèques auquel nous avons été peu préparés. D'autant plus que, les études d'Anderla le démontrent, la croissance quantitative, loin de s'arrêter, va progresser et s'accélérer pendant quelques années encore ${ }^{6}$. Cette explosion de l'information a pris au dépourvu non seulement les professionnels de l'édition mais aussi les spécialistes de la documentation qui sont restés sans solution valable, outre celle d'augmenter les budgets et les superficies en conséquence.

Autre élément qui assombrit le tableau et complique le dilemme auquel nous sommes confrontés, l'édition, qui croît à un rythme incontrôlable, n'est pas sans présenter des failles majeures au niveau du contenu. L'édition, selon John Dessauer dans une série de trois articles publiés dans Publishers Weekly, aurait atteint, elle aussi, son niveau d'incompétence?. Manuscrits imparfaits, redites, absence d'originalité, voilà autant de reproches qui sont formulés par l'auteur dans cette série d'articles. Sans prendre pour soi les multiples affirmations de Dessauer, il reste qu'il attache le grelot à un problème d'importance. Même en acceptant l'éclatement récent des disciplines scientifiques et l'arrivée massive de nouvelles disciplines, il apparaît peu probable que les champs de recherche soient suffisamment diversifiés pour assurer l'originalité de chaque volume. Tous et chacun répètent toujours un peu ce que d'autres ont déjà écrit. II ne faut donc pas s'étonner et considérer plutôt comme significatif le fait que la célèbre sentence "Publie ou périt», qui fut une cause première de l'explosion de l'information scientifique, soit en voie d'être remplacée par cette autre sentence plus exigeante "Soit cité ou périt».

D'autre part, les éditeurs, à cause des méthodes d'édition qu'ils utilisent, ont consacré eux-mêmes cette déficience de la qualité de l'information véhiculée. À titre d'exemple, il faut constater le nombre de périodiques et de livres parus, ces derniers temps, sur des sujets aussi à la mode que l'écologie et les problèmes d'énergie. Le point de vue commercial prend alors le dessus sur le point de vue scientifique et le résultat final ne va pas toujours dans le sens du progrès de la science.

5. Chiffres cités dans G. Anderla, L'information en 1985, Paris, OCDE, 1973 , p. 26.

6. Ibid., 31

7. John P. Dessauer, "Too many books?», Publishers Weekly, (September 7, 1974), 24-25; (October 7 , 1974), 34-35; (October 17, 1974), 32-33.
Conséquence immédiate de cette dévaluation de l'information: non seulement nos bibliothèques sont-elles encombrées par une masse documentaire impressionnante, mais encore commencent-elles à l'être par une information pas toujours originale et plus rapidement périmée.

Enfin, l'écart plus large entre l'offre et la demande qui existe dans les collections d'une certaine importance constitue un autre problème de taille pour nos bibliothèques. Or ces collections, à cause de la poussée de la production documentaire, ne sont plus réservées aux seules grandes institutions mais sont maintenant à la portée des moyennes et petites institutions. Les études de Trueswell en particulier ont démontré que si les documents contenus dans une bibliothèque ont un taux d'utilisation qui croît constamment dans les premières étapes du développement, ce taux a un accroissement nul ou minime une fois passé le stade d'une collection de base.

"We note, affirme Trueswell, that ap-
proximately 80 per cent of the circulation
requirements are satisfied by approxi-
mately 20 per cent of the library's hold-
ings... about 93 per cent of the circula-
tion was satisfied by approximately 50
per cent of the holdings." 8

En d'autres mots, il apparaît évident certaines analyses coût/avantage l'ont démontré - que la valeur relative de l'information véhiculée diminue constamment par rapport à ses usagers potentiels au fur et à mesure que les possibilités de choix augmentent. Le même livre dans une collection de 10 mille, 100 mille ou 1 million de volumes verra sa valeur décroître constamment parce qu'il est moins susceptible d'être utilisé. Face à ce problème, il faut se garder de conclure que la demande d'information diminue en fonction de l'augmentation de la collection. Elle a, au contraire, tendance à augmenter selon l'apparition de facteurs plus déterminants, tels une clientèle élargie, un accès plus facile à la documentation ou encore l'avènement de méthodes pédagogiques nouvelles. Cependant, un fait demeure: une bibliothèque de 500,000 livres n'a pas nécessairement plus de valeur qu'une bibliothèque de 100,000 livres puisque le phénomène de redondance $y$ est beaucoup plus fort. Or, la tendance naturelle va dans le sens d'un accroissement régulier des collections et non dans le sens inverse. Conséquence immédiate de cette situation:

8. R. C. Trueswell, "Some behavioral patterns of the library users: the $80 / 20$ rule». Wilson Library Bulletin, (January 1969), 458. 
passé un certain seuil, nos bibliothèques s'encombrent également d'une documentation dont la valeur globale n'est pas pleinement assumée.

\section{Une action qui s'impose}

Face à une certaine politique d'attentisme, tenant compte par ailleurs de cette pression d'une masse documentaire toujours plus imposante, souvent inégale dans son contenu et de plus en plus sous-utilisée, les professionnels de la documentation doivent agir avec promptitude. Ils se doivent d'inventer des systèmes et des méthodes qui sachent répondre à des problèmes nouveaux. La célébrité bibliothéconomique n'est plus réservée à celui qui mettra sur pied un nouveau système de classification, mais bien à celui qui réussira à développer un système de déclassification qui déterminerait, à l'avance, le moment d'éliminer un livre de la collection et le sort qu'on lui destine. Nous ne pouvons plus tellement compter sur des catastrophes naturelles ou des événements extérieurs pour nous sortir d'embarras en cette matière. Les guerres sont moins fréquentes et les bibliothèques, à ce que nous sachions, ne sont pas considérées comme des objectifs stratégiques, ce qui élimine les possibilités de bombardement. Les édifices sont aussi mieux construits, ce qui diminue considérablement les risques d'incendies. Des systèmes anti-vol de plus en plus raffinés ont réduit sensiblement les fuites régulières qui permettaient un élagage naturel. Il y a toujours une possible pénurie de matières premières qui réglerait le problème à la source pour les prochaines années, mais c'est là une solution qui présente l'inconvénient de ne pas régler le problème de fond.

Ce qu'il faut, ce qui est urgent à plus d'un point de vue, c'est d'assurer, par le moyen de l'élagage, le dynamisme de nos collections de bibliothèques plutôt que de perpétuer une inertie dommageable. Ce qui s'avère nécessaire, c'est d'insérer de plus en plus, dans nos politiques et nos procédures, certaines méthodes de travail propres à cette fonction. Agir ainsi va peut-être à l'encontre d'une longue tradition de conservation, mais correspond davantage à des objectifs d'enseignement et de recherche et à l'idée que nous nous faisons d'une collection toujours en devenir.

\section{Certaines solutions trompeuses}

On a souvent tendance, en ce domaine, à se contenter de ce que nous qualifierions de leurres ou, tout au moins, de demi-solutions. Parmi ces demi-solutions les plus souvent mises de l'avant, nous comptons la microreproduction et l'entreposage commun. Les deux se caractérisent par le fait qu'ils ne font, somme toute, que déplacer le problème, le premier en versant une documentation inutilisée sur un support différent, le second en déménageant une partie de ses collections dans des locaux différents.

Pour un, l'entreposage commun demeure une solution intéressante au problème d'espace que connaissent les grandes bibliothèques. Il permet de conserver une documentation encore utile à un coût moins prohibitif. Cependant, il en va tout autrement lorsque l'entrepôt commun est utilisé pour conserver une documentation périmée ou en voie de l'être. II n'est alors rien de moins qu'une solution de facilité. Plutôt que d'éliminer complètement une documentation devenue encombrante en l'échangeant, en la vendant ou simplement en la donnant, on choisit de la conserver quelque part, le plus loin possible, pour ne pas occasionner trop de maux de tête, quitte à revenir sur sa décision si nécessaire. Dans cette optique, l'engorgement, au lieu de se faire à l'intérieur de la bibliothèque, se fait à l'extérieur. De plus, l'entreposage commun, vu sous cet angle, demeure une solution onéreuse si l'on considère d'une part l'investissement requis et d'autre part le taux d'utilisation prévu. C'est payer bien cher un tranquillisant. On pourrait, par ailleurs, formuler les mêmes remarques au sujet du rayonnage compact, très utile pour régler les problèmes d'espace mais moins de mise pour solutionner les problèmes posés par la présence d'une documentation devenue inutile.

La microreproduction ne peut pas davantage être envisagée comme une solution valable. Outre le fait qu'elle ne permet pas l'élagage mais tout au plus la miniaturisation de l'information, elle ne peut s'appliquer à tous les types de documents. Sont exclus, en effet, d'une opération de microreproduction tous les ouvrages de référence, les volumes illustrés, les manuels, etc. De plus, il s'agit là d'une entreprise trop coûteuse en personnel et en matériel et assujettie à trop de contraintes pour une documentation périmée et sans valeur. C'est également une entreprise superflue pour une documentation souvent consultée. Elle ne permet pas alors de récupérer les superficies libérées par la transformation de l'imprimé en microreproduction, superficies qui doivent être utilisées pour le mobilier, l'équipement et le personnel requis pour son exploitation. Donc, comme l'affirme F. C. Lynden, dans un article intéressant publié dans Microform Review: 
"A microform conversion project should not be regarded as a panacea; in order to insure user acceptance, cost savings and quality control, a good program requires a careful, analytical study of all the benefits and problems associated with using microforms in place of hard copy."

II convient cependant de considérer le microdocument comme palliatif acceptable pour une documentation indispensable mais utilisée moins fréquemment, ce qui est le cas, notamment, des périodiques et des thèses.

\section{Quelques conditions de réussite}

Si donc la microreproduction et l'entreposage commun ne peuvent être considérés comme des moyens suffisants pour régler l'épineux problème soulevé au départ, que reste-t-il comme proposition recevable? Nous croyons inutile, ici, de proposer des méthodes particulières de gestion de la documentation propres à l'élagage. Nous ne pensons pas que le cadre de cet article soit le lieu privilégié pour traiter de cet aspect. Nous croyons cependant opportun de souligner à nouveau que ces procédures sont possibles, qu'elles existent déjà dans quelques institutions et qu'elles deviendront vite essentielles au bon fonctionnement des bibliothèques d'enseignement collégial et universitaire et des bibliothèques de recherche. Une partie importante de la documentation, nous le répétons, devra tôt ou tard être éliminée de la collection selon un processus normal et régulier. Et cela devra se faire aussi normalement et aussi régulièrement que la circulation du document ou l'indexation de son contenu. C'est toujours dans cet esprit que nous parlons de l'élagage des collections. Et pour être bien certain que cette opération soit une opération réussie, il est nécessaire d'appuyer ses assises sur deux conditions fondamentales: un aménagement administratif à l'intérieur même de la bibliothèque et, à l'extérieur, une collaboration de la part des utilisateurs.

\section{Un aménagement administratif}

L'élagage des collections de la bibliothèque, il ne faut pas se le cacher, est coûteux à plusieurs points de vue. II suppose un réaménagement administratif et budgétaire absolument essentiel dans ce contexte. II serait oiseux, pour un administrateur, de recon-

9. F. C. Lynden, "Remplacement of hard copy by microforms", Microform Review, vol. 4, no. 1 (January 1975), 22. naître l'importance et l'enjeu de cette fonction si, par ailleurs, il ne fournit pas les éléments requis pour mettre sur pied et entretenir un tel système. Si on comprend l'élagage dans le sens de l'élimination définitive de la collection des documents impertinents, il faut bien se rendre compte que cela implique qu'il faille $y$ affecter un personnel adéquat et un budget spécifique. Déterminer qu'un volume n'est plus utile dans la collection, compte tenu de la transformation des programmes d'enseignement ou de recherche, de l'évolution de la discipline ou encore de sa valeur intrinsèque, voilà une tâche vraiment professionnelle. Elle demande autant de connaissances, de perspicacité et de justesse d'appréciation que le choix ou le traitement de la documentation. Elle exige d'être soutenue par un personnel d'appoint qui soit en mesure d'accomplir les tâches techniques connexes à cette fonction. Dans les petites bibliothèques, ces diverses tâches devront être accomplies par le personnel régulier, particulièrement par celui déjà affecté au développement et à l'exploitation de la collection. Ce n'est pas là un obstacle au succès de l'opération, à la condition que le partage des tâches soit clairement défini.

Dans ce contexte d'un réaménagement administratif, il est important d'instaurer, pour soutenir cette fonction, un instrument de gestion considéré essentiel en management et dont l'établissement produit généralement d'excellents résultats. Un «système d'information de gestion" (MIS), grâce à une saisie de données précises, à leur traitement et à leur exploitation, permet en effet aux responsables administratifs de prendre des décisions à la lumière d'une information suffisante.

«il répond, affirme William Bolchino, aux besoins des dirigeants en matière de données précises, opportunes et intéressantes pour planifier, analyser et contrôler les activités de l'organisation, et en optimiser aussi la survie et l'expansion. Le système d'information de gestion fournit à la direction les moyens d'entrée, de traitement et de sortie des données, ainsi qu'un réseau de décision avec retour des informations, afin de l'aider à s'adapter aux changements en cours et à venir de l'environnement intérieur et extérieur de l'organisation.»" ${ }^{10}$

Appliqué aux bibliothèques, un tel système recouvre les statistiques habituelles de fréquentation, de prêt, de classement, d'acquisition, mais également, avec l'avènement de

10. William Bolchino, Systèmes d'information pour le management, Paris, Publi-Union, 1972, p. 17. 
l'automatisation, peut fournir les données sur les coûts de traitement, de conservation et d'exploitation, sur la force ou la faiblesse des divers secteurs de la collection et sur les besoins à combler (programmes d'enseignement, projets de recherche, clientèles étudiantes, etc.). II permet également certaines analyses statistiques ainsi que des analyses de corrélation. Ces différentes informations existent déjà à l'état embryonnaire dans la plupart des bibliothèques, mais sont trop souvent incomplètes ou mal utilisées. Or la planification, l'analyse et le contrôle des opérations d'évaluation et d'élagage ne peuvent être en effet assurés des conditions minimales de succès si ces actions ne sont pas entourées d'une connaissance suffisante des diverses données qui sont requises et qui facilitent l'application des critères de décision. Ce sont de telles données significatives qui seront obtenues par l'instauration d'un bon «système d'information de gestion".

Cette fonction d'élagage, comme nous pouvons le constater, n'est donc pas une simple opération de nettoyage saisonnier. Elle exige qu'on y affecte les ressources humaines et budgétaires appropriées. Mais agir dans ce domaine en n'accordant pas les moyens nécessaires à la réussite de cette entreprise ne saurait être autre chose qu'un exercice de style ou une activité du dimanche.

\section{Son acceptation par les usagers}

Les inquiétudes manifestées par les usagers, les professeurs plus particulièrement, constituent un obstacle majeur qu'il faut réussir à surmonter sous peine de provoquer une incompréhension capable de mettre en cause les objectifs visés. Cette réticence à voir retirer de la collection une documentation peut-être susceptible de servir à nouveau est généralement aussi forte que la pression exercée sur les responsables de la bibliothèque pour acheter une documentation jugée indispensable. En effet, autant il est hasardeux de développer une collection de bibliothèque sans, au préalable, obtenir la participation des professeurs impliqués, autant peut être vouée à l'échec une démarche d'évaluation et d'élagage qui n'aurait pas été acceptée par ces responsables de la dimension pédagogique. II convient donc d'accorder à ce facteur une importance toute particulière.

II faut comprendre que nos bibliothèques, aussi bien que les lecteurs qui les fréquentent, ont été formés à l'enseigne du "tout sur place». Les moyens de communication n'ayant pas toujours été ce qu'ils sont actuellement, la réaction normale des individus était alors de ne pas croire à la possibilité de profiter des ressources possédées par d'autres bibliothèques facilement accessibles, mais plutôt d'exiger d'avoir sur place une documentation souvent plus qu'abondante. Ce besoin d'avoir sur place toute la documentation utile à l'enseignement et à la recherche est encore très présent dans l'esprit de tous et chacun. Aucune restriction budgétaire, aucune poussée inflationniste du coût de la documentation ne pourraient avoir définitivement raison de ce sentiment. Récemment encore, un département d'une nouvelle université québécoise ne définissait-il pas ses besoins documentaires à partir de l'hypothèse que la quantité de volumes possédée par les autres universités est un bon indicateur de ce que sa propre bibliothèque devrait avoir? Aussi, en raison de ce sentiment, voit-on émerger spontanément cette résistance de la part des enseignants à toute velléité de mise à jour unilatérale d'une collection de bibliothèque, résistance qui pourra aller de la simple critique à l'affrontement brutal et qui ne sera pas toujours empreinte de l'objectivité nécessaire. C'est quasiment un réflexe de défense.

Pour faire passer cette catégorie d'usagers du stade du refus à celui de l'acceptation, il faut savoir user de prudence et abuser d'information. II faut multiplier les démarches de consultation et bien expliquer les motifs qui sous-tendent une telle action. II faut également être en mesure de démontrer rationnellement que, loin d'appauvrir la collection, un élagage régulier permet son enrichissement et maximise sa valeur. II faut enfin garantir l'accessibilité éventuelle aux documents élagués par l'intervention de moyens extérieurs tel le recours au prêt entre bibliothèques. II peut paraître superflu de s'entourer ainsi d'autant de précautions, mais c'est pourtant là une condition importante de réussite.

Ces deux éléments, donc, à savoir un réaménagement administratif et budgétaire et une acceptation de fait de la part des usagers devraient assurer le succès d'une opération qui montre bien, comme l'affirmait Gérard Mercure dans son article sur les «Média documentaires," que "le temps est déjà révolu où l'on appréciait la qualité d'une bibliothèque à son nombre de volumes et de périodiques mais qu'il faut dorénavant l'évaluer à la diversité des services auxquels elle est reliée ou abonnée et à la qualité de la documentation qu'elle diffuse »11.

11. Gérard Mercure, "Les média documentaires", Documentation et bibliothèques, vol. XX, no 3 (septembre 1974), 140. 


\section{Une solution à la source}

De toute évidence, le fait de donner à l'élagage les lettres de noblesse que la bibliothéconomie lui a refusées jusqu'à maintenant ouvre la porte à une amélioration qualitative des collections de bibliothèques. Mais ce ne sera toujours qu'une solution partielle, et en partie insatisfaisante, aux problèmes que nous soulevions en première partie. Elle doit être accompagnée d'une autre solution, plus globale celle-là, qui saura apporter des remèdes permanents à un problème permanent. Tout comme il est utopique de croire qu'une bibliothèque peut acquérir aujourd'hui toute la documentation reliée aux champs d'intérêt de ses usagers, il est également illusoire de croire que la même bibliothèque ne peut se départir d'une partie de la documentation amassée au rythme des saisons et des aléas budgétaires. Or cette exhaustivité dans le choix comme cette parcimonie dans l'élagage ne poseront plus autant de problèmes à nos spécialistes de la documentation le jour où ils seront assurés que les bibliothèques nationales seront en mesure de répertorier, conserver et diffuser la totalité de la production documentaire nationale.

Cet aspect de la conservation et de la confection de répertoires bibliographiques est inclus dans les textes régissant la plupart des bibliothèques nationales. De plus, l'obligation du dépôt légal vient souvent renforcer cette dimension de la conservation. Aussi, peut-on penser qu'avec la généralisation de ces institutions on aura rapidement atteint un certain contrôle international de la documentation. Si, par ailleurs, on tient compte de l'existence de services spécialisés ou de "centrales de regroupement de l'information" qui remplissent déjà une fonction de collecte et de conservation d'une documentation plus spécifique non publiée par le circuit commercial habituel, on $a$, de ce fait, la presque assurance que la pérennité de l'information sera sauvegardée. Ce qui ajoute à l'intérêt de cette solution, c'est que la documentation est conservée sur une base rationnelle par ceux-là même qui ont comme rôle premier de le faire. II n'est alors plus question pour les autres bibliothèques de suppléer à ces institutions et de vouloir tout conserver. Elles pourront ainsi se satisfaire de ne conserver que le meilleur.

Cette dimension de la conservation n'est toutefois recevable que si elle est complétée d'une autre dimension toute aussi essentielle, celle de la diffusion. II ne saurait, en effet, être question de faire reposer sur des organismes extérieurs une partie de ses besoins documentaires, si ces derniers ne peuvent rendre disponible, dans un délai raisonnable et sur un support acceptable, la documentation qu'ils possèdent. Cette accessibilité à l'information est acquise dans le cas des "centrales de regroupement de l'information" (de type ERIC ou NTIS) qui sont axées autour de la notion même de diffusion. Elle est cependant moins garantie dans le cas des bibliothèques spécialisées et des bibliothèques nationales. Les services traditionnels de prêt entre bibliothèques sont alors trop complexes, plutôt lents et excluent généralement toute une documentation qui, justement, devrait être diffusée sans restriction. Il y a donc là des correctifs importants à apporter et qui ne seront satisfaisants qu'au moment où ils permettront aux diverses bibliothèques d'avoir un accès facile à cette documentation. Ce qui importe alors, ce n'est pas tant de permettre l'accès instantané par téléfacsimilé ou autrement, bien que les délais de livraison soient alors un élément important à considérer; ce qui importe vraiment, c'est que les bibliothèques qui ont un rôle de conservation assurent, dans les différents points d'utilisation, l'accessibilité du document dans sa version originale ou en fac-similé par la microcopie ou la photocopie. Le prix à payer sera probablement élevé compte tenu des droits d'auteur, de la manutention et de la transmission du document, mais ce sera encore moins onéreux que de conserver une collection qui n'est, somme toute, qu'une collection d'appoint.

Chaque bibliothèque d'enseignement et de recherche pourra alors se payer ce petit luxe de planifier le développement de ses collections en fonction de ce qui est disponible ailleurs et non plus en fonction d'un isolement qui porte à l'autarcie. Peut-être faudra-t-il quelques années encore avant d'en arriver à cette nouvelle génération de bibliothèques! Mais à ce moment nous ne serons plus devant un choix à faire: cette bibliothèque nous aura déjà été imposée.

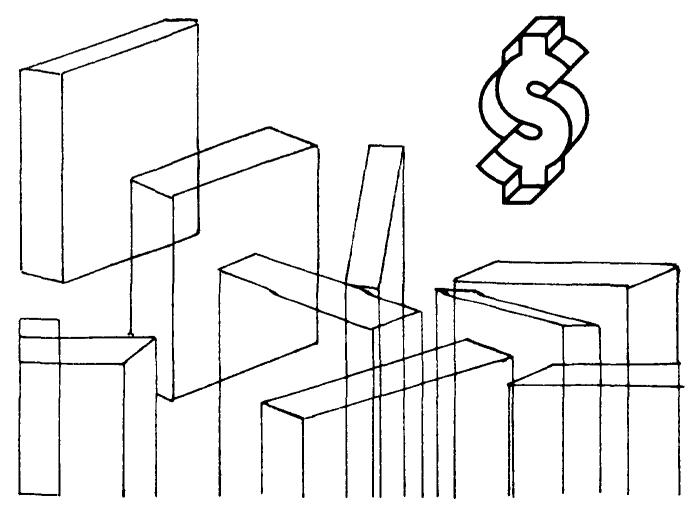

